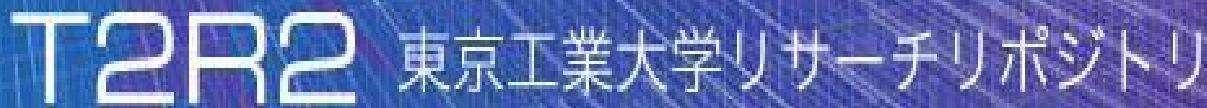

\section{Tokyo Tech Research Repository}

\section{論文 /著書情報 \\ Article /Book Information}

\begin{tabular}{|c|c|}
\hline Title & $\begin{array}{l}\text { Multispectral image compression for spectral and color reproduction } \\
\text { based on lossy to lossless coding }\end{array}$ \\
\hline Authors & $\begin{array}{l}\text { Kazuma Shinoda, Yuri Murakami, Masahiro Yamaguchi, Nagaaki } \\
\text { O hyama }\end{array}$ \\
\hline Citation(English) & Proceedings of SPIE, Vol. $7532, \quad$, pp. $75320 \mathrm{H}$ \\
\hline 発行日 /Pub. date & 2010,1 \\
\hline DOI & $10.1117 / 12.838843$ \\
\hline 権利情報 /Copyright & $\begin{array}{l}\text { 本著作物の著作権はSociety of Photo-O ptical Instrumentation } \\
\text { Engineersに帰属します。 } \\
\text { Copyright } 2010 \text { Society of Photo-O ptical Instrumentation Engineers. } \\
\text { One print or electronic copy may be made for personal use only. } \\
\text { Systematic reproduction and distribution, duplication of any material in } \\
\text { this paper for a fee or for commercial purposes, or modification of the } \\
\text { content of the paper are prohibited. }\end{array}$ \\
\hline
\end{tabular}




\title{
Multispectral image compression for spectral and color reproduction based on lossy to lossless coding
}

\author{
Kazuma Shinoda $^{a^{*}}$, Yuri Murakami ${ }^{b}$, Masahiro Yamaguchi $^{b}$ and Nagaaki Ohyama ${ }^{b}$ \\ ${ }^{a}$ Interdisciplinary Graduate School of Science and Engineering, Tokyo Institute of Technology, \\ Yokohama, Japan; \\ ${ }^{b}$ Imaging Science \& Engineering Laboratory, Tokyo Institute of Technology, Yokohama, Japan;
}

\begin{abstract}
In this paper we propose a multispectral image compression based on lossy to lossless coding, suitable for both spectral and color reproduction. The proposed method divides a multispectral image data into two groups, RGB and residual. The RGB component is extracted from the multispectral image, for example, by using the XYZ Color Matching Functions, a color conversion matrix, and a gamma curve. The original multispectral image is estimated from RGB data encoder, and the difference between the original and the estimated multispectral images, referred as a residual component in this paper, is calculated in the encoder. Then the RGB and the residual components are encoded by JPEG2000, respectively a progressive decoding is possible from the losslessly encoded code-stream. Experimental results show that, although the proposed method is slightly inferior to JPEG2000 with a multicomponent transform in rate-distortion plot of the spectrum domain at low bit rate, a decoded RGB image shows high quality at low bit rate with primary encoding of the RGB component. Its lossless compression ratio is close to that of JPEG2000 with the integer KLT.
\end{abstract}

Keywords: Multispectral image compression, lossy and lossless compression, JPEG2000, color reproduction, sRGB

\section{INTRODUCTION}

Multispectral images have been used mostly in remote sensing, but are also being extended to fields requiring high fidelity color reproductions such as tele-medicine and digital archive of artworks. ${ }^{1}$ These multispectral imaging systems are valued because they offer improved color reproduction quality under arbitrary illuminant, as well as the two-dimensional spectral information useful for the image analysis. In response to the huge volume of multispectral images, many compression algorithms have been developed for efficient transmission and storage; yet most of these are in the field of remote sensing, and aim to preserve spectral accuracy and minimize the difference between the original and the reconstructed multispectral images. In, ${ }^{2}$ that uses KarhunenLoeve transform (KLT) as the spectral transform, followed by two-dimensional wavelet transform as the spatial compression scheme. An anisotropic 3D wavelet transform is also applied to the multispectral data. ${ }^{3,4}$ However, in the applications that require high colorimetric accuracy, it is of vital importance to keep the difference between the original and reconstructed color images small.

A significant contribution to multispectral imaging system was made by Keusen ${ }^{5}$ and Hill. ${ }^{6}$ In the proposed architecture, the spectral stimulus is expanded by a set of basis functions, and encoded as $K$-channel coefficients for the basis functions. The first three components of the encoded data correspond to the tristimulus values under a standard illuminant, and the remaining $K-3$ channels represent invisible spectral components. As a multispectral image compression technique considering the colorimetric accuracy, a modified Karhunen-Lueve (KL) transform called weighted KL transform ${ }^{7}$ was proposed and combined with the JPEG2000 scheme. ${ }^{8}$ Labplus $^{9}$ has also been proposed as a method of based on the colorimetric accuracy. Labplus transforms the first three channel into CIE $L^{*} a^{*} b^{*}$ color space by nonlinear transform. For video compression, three methods that support multichannel images, MPEG4 studio profile, H.264/AVC, and Motion JPEG2000, were tested in. ${ }^{10}$ The test included a coding method in which a multispectral image was converted into visible and invisible components,

*E-mail: shinoda.k.ad@m.titech.ac.jp

Image Processing: Algorithms and Systems VIII, edited by Jaakko T. Astola, Karen O. Egiazarian, Proc. of SPIE-IS\&T Electronic Imaging, SPIE Vol. 7532, 75320H · @ 2010 SPIE-IS\&T

CCC code: $0277-786 \mathrm{X} / 10 / \$ 18 \cdot$ doi: $10.1117 / 12.838843$ 


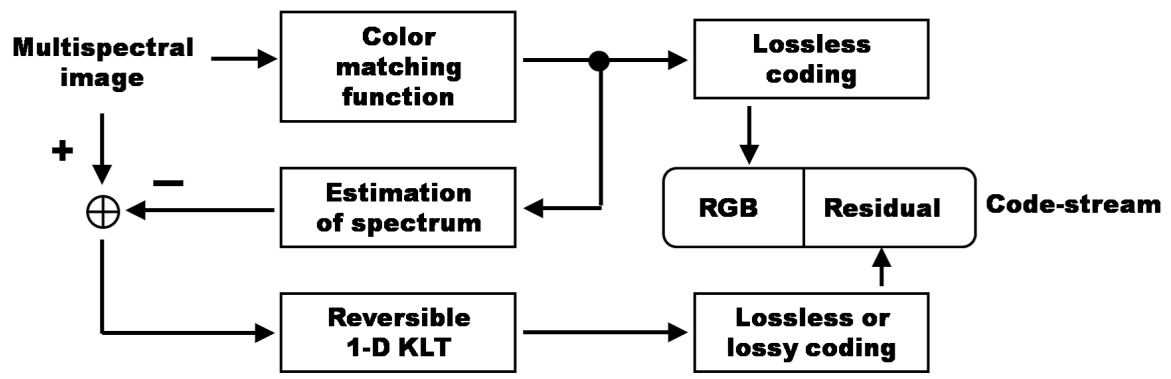

Figure 1. Outline of the coding method.

with the subsampling of chrominance signals. These methods are valuable for color reproduction applications, whose objective is to reduce the error in both color images and multispectral images under low bit rates.

This paper presents a lossy to lossless compression for multispectral image, while keeping the quality of RGB image, which affords high-fidelity colorimetric reproduction. The whole point of our method is that a conventional RGB image appears early in a code-stream. As the RGB format, the sRGB ${ }^{11}$ colorspace (compatible with ITUR BT.709 as well) is used in this paper. A multispectral image is divided into two groups, RGB component and residual component, and these components are encoded by a seamless coding independently. The seamless method is based on wavelet-based coding with JPEG2000. The proposed method encodes the RGB component primary, this approach allows us to get high quality RGB image and not so much distortion in the spectrum domain.

\section{CODING ALGORITHM}

\subsection{Calculation of RGB from MSI}

The coding algorithm is illustrated in Fig. 1. The RGB component is calculated from the original multispectral image (MSI) by using the CIE XYZ Color Matching Functions under the standard illuminant of D65. Before the calculation of XYZ, we estimate a real spectral refrectance from the multispectral data for natural color reproduction. The spectral reflectance $f$ is obtained by Wiener estimation from the original multispectral data $\boldsymbol{g}$, where the spectral sensitivity of the multispectral camera is used. A N-dimensional column vector $\boldsymbol{g}$ represents a multispectral data, and a L-dimensional column vector $f$ represents a spectral reflectance, where $\mathrm{N}$ is the number of filters in multispectral camera, and $\mathrm{L}$ is the number of wavelength samples. The magnitude relation between $N$ and $L$ is $N<L$, let $N=16, L=401$ in this paper.

The transform coefficients $\boldsymbol{g}$ of $\boldsymbol{f}$ is

$$
g=H f
$$

where $\boldsymbol{H}$ is a system matrix of a camera sensor. An estimated $\hat{\boldsymbol{f}}$ is

$$
\hat{f}=M g,
$$

where $\boldsymbol{M}$ is a $L \times N$ matrix. We use a Wiener matrix for $\boldsymbol{M}$, which is described as

$$
\boldsymbol{M}=\boldsymbol{R} \boldsymbol{H}^{T}\left(\boldsymbol{H} \boldsymbol{R} \boldsymbol{H}^{T}+\boldsymbol{N}\right)^{-1},
$$

where $\boldsymbol{R}$ is a first-order Markov model of $L \times L$ matrix, $\boldsymbol{H}$ is a system model of $N \times L$ matrix, and $\boldsymbol{N}$ is a $L \times L$ noise matrix. The spectral spectral reflectance of natural objects can be well approximated by limited number 
of basis functions. Therefore, first-order Markov model $\boldsymbol{R}$ has been used to approximate $\left\langle\boldsymbol{f} \boldsymbol{f}^{T}\right\rangle$ :

$$
\boldsymbol{R}=\left[\begin{array}{ccccc}
1 & \rho & \rho^{2} & \ldots & \rho^{L-1} \\
\rho & 1 & \rho & \ldots & \rho^{L-2} \\
\rho^{2} & \rho & 1 & \ldots & \rho^{L-3} \\
\vdots & \vdots & \vdots & \ddots & \vdots \\
\rho^{L-1} & \rho^{L-2} & \rho^{L-3} & \ldots & 1
\end{array}\right]
$$

where $\rho$ indicates the correlation between neghboring bands. It can well aproximate the covariance matrix of the original spectral reflectance when $0.95<\rho<1 .^{12}$

A three-dimensional column vector $\boldsymbol{X}$ represents a XYZ tri-stimulus as

$$
\begin{aligned}
\boldsymbol{X} & =\boldsymbol{T E \hat { f }} \\
& =\boldsymbol{T E M g}
\end{aligned}
$$

where $\boldsymbol{T}$ is a $3 \times L$ matrix of the XYZ color matching function, $\boldsymbol{E}$ is a $L \times L$ diagonal matrix of the spectral power distribution of an illumination. Let $\boldsymbol{H}^{\prime}=\boldsymbol{T} \boldsymbol{E} \boldsymbol{M}$ be a forward color-matching-function.

For the RGB color space gamma, we use generic sRGB profiles and an ideal gamma as

$$
\begin{gathered}
{\left[\begin{array}{l}
R^{\prime} \\
G^{\prime} \\
B^{\prime}
\end{array}\right]=\left[\begin{array}{ccc}
3.2410 & -1.5374 & -0.4986 \\
-0.9692 & 1.8760 & 0.0416 \\
0.0556 & -0.2040 & 1.0570
\end{array}\right]\left[\begin{array}{c}
X \\
Y \\
Z
\end{array}\right]} \\
{\left[\begin{array}{l}
R \\
G \\
B
\end{array}\right]=\left[\begin{array}{c}
\operatorname{ROUND}\left(R^{1 / 2.2}\right) \\
\operatorname{ROUND}\left(G^{1 / 2.2}\right) \\
\operatorname{ROUND}\left(B^{1 / 2.2}\right)
\end{array}\right],}
\end{gathered}
$$

where, R, G and B are clipped into [0,255]. The obtained RGB image is encoded loslessly with JPEG2000, which includes $\mathrm{YCbCr}$ color conversion.

\subsection{Estimation of MSI from RGB}

For the estimation of the original spectral image from the RGB values, we use Wiener estimation pixel by pixel. $\quad \boldsymbol{X}^{\prime}$ is calculated from the inverse operation of Equation (7) and Equation (8). The estimated original multispectral data $\hat{\boldsymbol{g}}$ is described as

$$
\hat{\boldsymbol{g}}=\overline{\boldsymbol{g}}+\boldsymbol{S}\left(\boldsymbol{X}^{\prime}-\boldsymbol{H}^{\prime} \overline{\boldsymbol{g}}\right),
$$

where $\overline{\boldsymbol{g}}$ is a mean vector of $\boldsymbol{g} . \boldsymbol{S}$ is a $N \times 3$ matrix, which is

$$
\begin{aligned}
\boldsymbol{S} & =(\boldsymbol{T E} \boldsymbol{M})^{+} \\
& =\boldsymbol{H}^{\prime+}
\end{aligned}
$$

Derivation of the matrix $\boldsymbol{S}$ is also ill-conditioned like that of $\boldsymbol{M}$, and here a Wiener estimation for $\boldsymbol{S}$ is given by

$$
\boldsymbol{S}=\boldsymbol{C}_{g} \boldsymbol{H}^{\prime T}\left(\boldsymbol{H}^{\prime} \boldsymbol{C}_{g} \boldsymbol{H}^{\prime T}\right)^{-1}
$$

where $\boldsymbol{C}_{g}$ is a variance-covariance matrix of $\boldsymbol{g}$. 


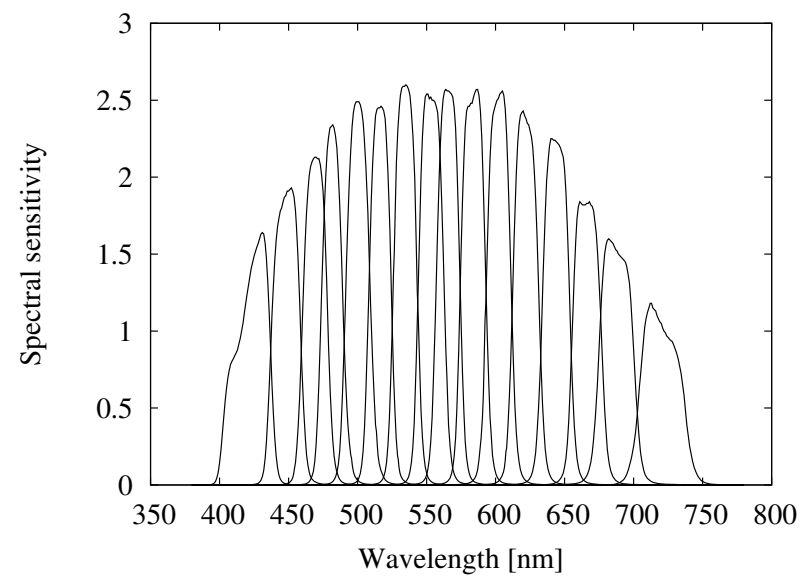

Figure 2. Spectral sensitivities of the 16-band multispectral camera used in experiments.

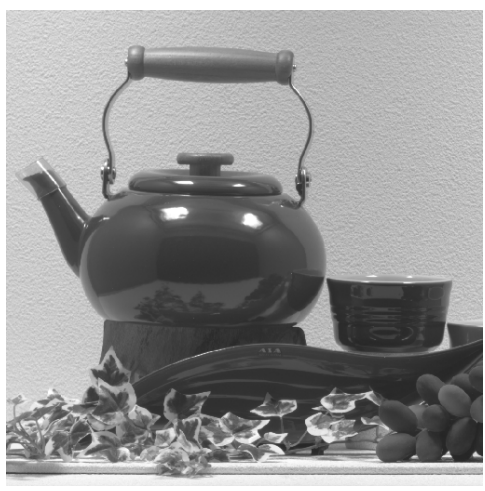

(a) Image 01

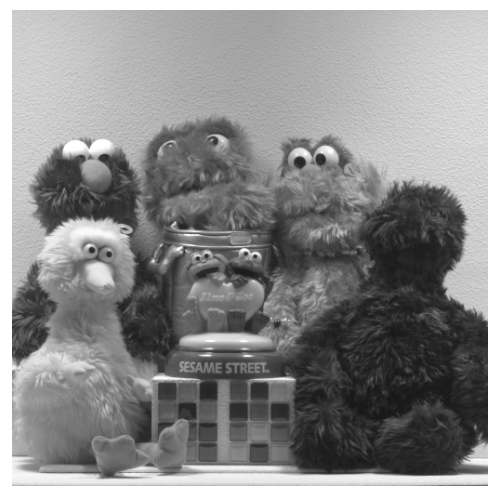

(b) Image 02

Figure 3. Test images. 512x512, 16 bands, 16 bpppb (Bits / pixel / band).

\subsection{Coding of Residual}

For further encoding of the spectral information, the spectral contribution by the RGB components is subtracted from the original multispectral image. We regard this difference as a residual; $\boldsymbol{g}-\hat{\boldsymbol{g}}$. Note that the residual consists of the same number of bands as the original. For the decorrelation in the wavelength axis of residual data, we use a reversible Karhunen-Loeve transform as the spectral transform, which is based on a single-row elementary reversible matrix (SERM) factorization. ${ }^{13}$ A variance-covariance matrix of the original multispectral image is obtained in advance.

The coding block in Fig. 1 means to apply a seamless coding, each of the residual channel, which is obtained by reversible KLT, is encoded by JPEG2000. The first RGB values is losslessly compressed, while the residual values can be compressed from lossy to lossless. A progressive decoding is equipped with the losslessly encoded code-stream. The decoding scheme is the reverse procedure of the encoding.

\section{EXPERIMENTAL RESULTS}

For the following results, we use a multispectral camera with 16 narrow band color filters as shown in Fig. 2. Fig. 3 shows test images that consists of $512 \times 512$ pixels with 16 -bit dynamic resolution. The RGB image is encoded by LuraWave ${ }^{14}$ software with LRCP (Layer, Resolution, Component, Position) progressive ordering. Each of the residual channel of the proposed method is encoded as a grayscale image with JPEG2000. JPEG2000 with a multicomponent transform (JPEG2000-MCT) is referred as a comparison. We use a matrix of reversible 
Table 1. Comparison of lossless bit rates in bpppb.

\begin{tabular}{|c|c|c|c|c|}
\hline & \multicolumn{2}{|c|}{ Spectrum } & \multicolumn{2}{c|}{ RGB } \\
\cline { 2 - 5 } & Proposed & JPEG2000 with MCT & Proposed & JPEG2000 with MCT \\
\hline Image 01 & 7.179 & $\underline{6.820}$ & $\underline{0.784}$ & 6.820 \\
\hline Image 02 & 6.234 & $\underline{5.947}$ & $\underline{0.774}$ & 5.947 \\
\hline
\end{tabular}

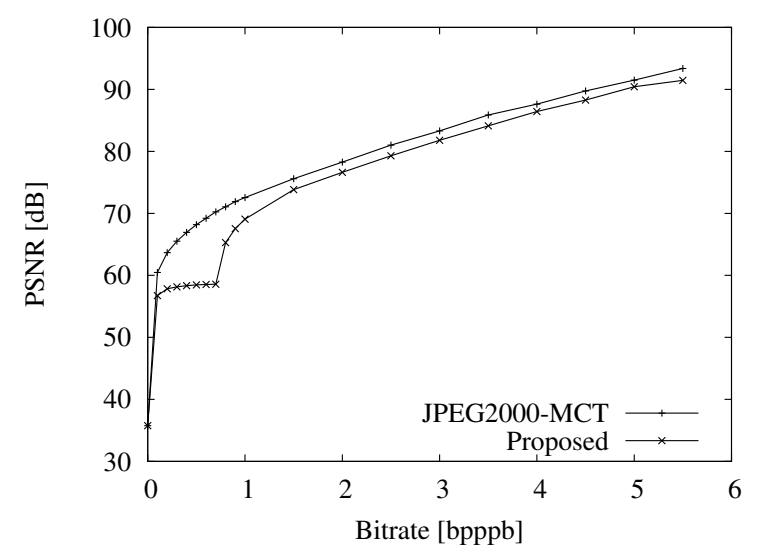

(a) Image 01

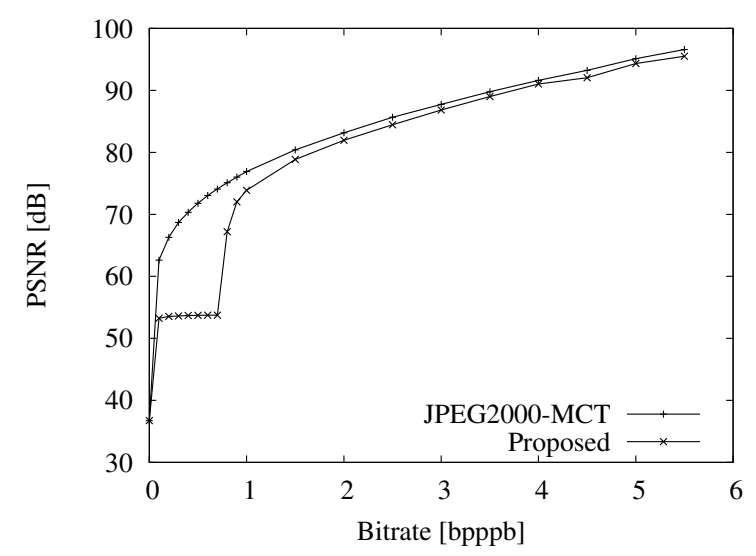

(b) Image 02

Figure 4. Lossy bit rates vs. PSNR on Spectral data.

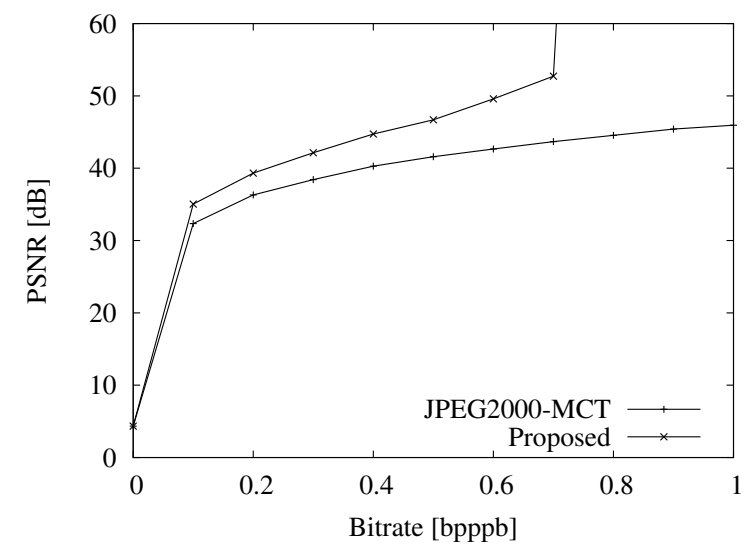

(a) Image 01

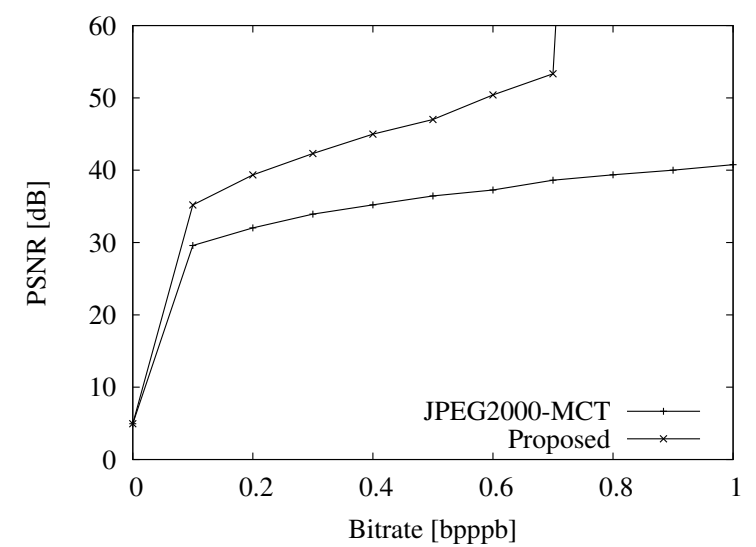

(b) Image 02

Figure 5. Lossy bit rates vs. PSNR on RGB data.

KLT for the multicomponent transform and LuraWave with LRCP progressive ordering for the bit-encoding. The proposal and the JPEG2000-MCT are applied with a lifting computation of the 5/3-tap reversible wavelet transform in the spatial domain. The estimation matrix and the reversible KLT require an additional information for decoding, but the additional code will be negligible in this paper because the bit rate amounts to only under 0.001 bpppb.

\subsection{Performance of Simple Decoding}

Fig. 4 shows a rate-distortion plot against bit rate on the spectrum domain. Although the proposed method is inferior to the JPEG2000-MCT in the low bit rate, the proposed method is competitive to the JPEG2000-MCT in the high bit rate. The number one reason is probably that the proposed method encodes RGB components 


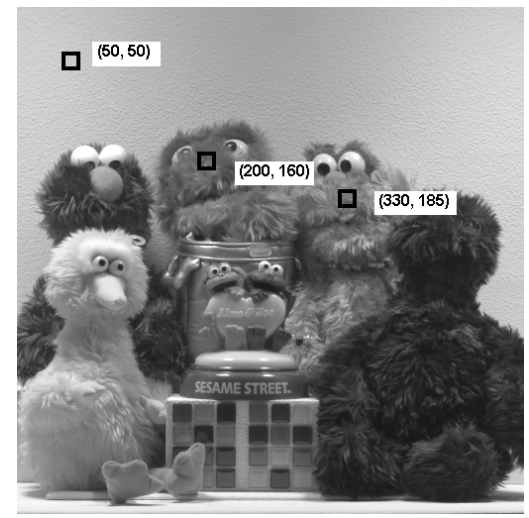

(a) Sampling points to check the reconstructed MSI

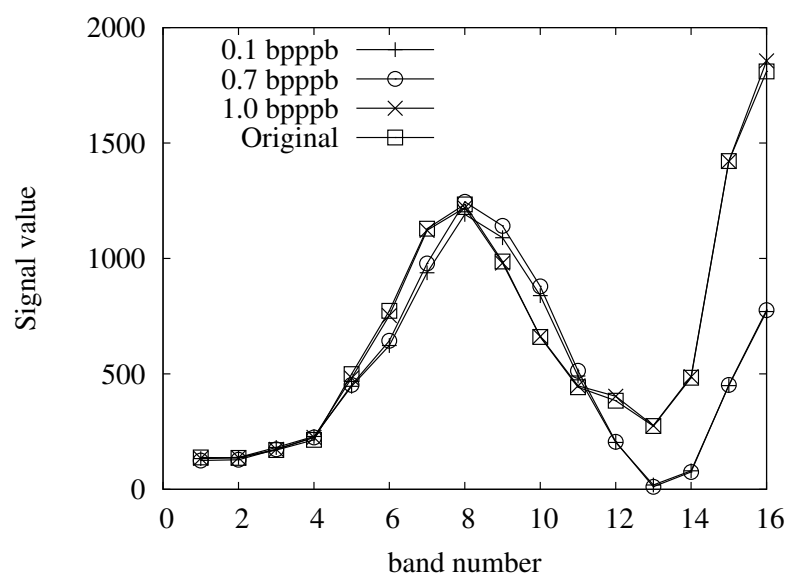

(c) Green $(200,160)$

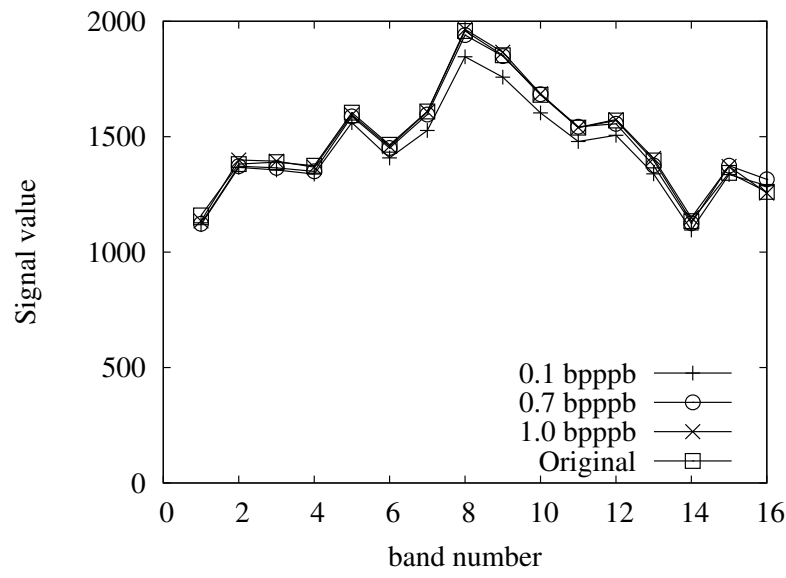

(b) Background $(50,50)$

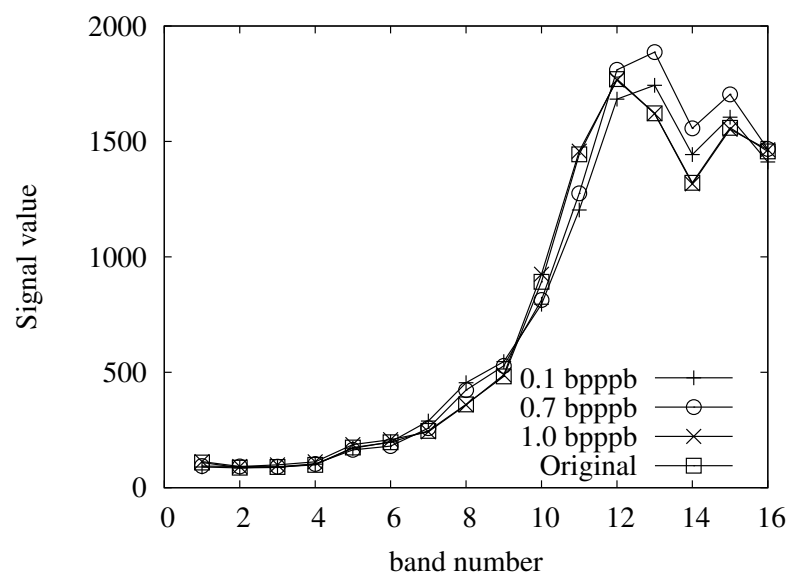

(d) Orange $(330,185)$

Figure 6. Decoded multispectral data at each points.

first of all and then moves into the coding of residual components after finishing the coding of RGB components. The sharp rise in the graph means a boundary between RGB and residual code-stream because the code-stream of less than 0.8 bpppb consists of only RGB components as shown in Table 1 . The graph suggested that RGB components can not contribute greatly to reduce the distortion in the spectrum domain. It would appear that 3 bands are not enough number of bands to estimate 16 bands in the low bit rate. We would like to focus on the resolution of this problem in the next section. On the other hand, the sharp rise at 0.7 to 0.8 bpppb is also considered to imply an effect of the primary component of the residual data. The significant residual data can be said to reduce the distortion rather than the insignificant RGB data.

In Table 1, the performance of the proposed method at lossless bit rate is inferior as compared with that of the JPEG2000-MCT. The original multispectral image is divided into RGB components and residual components in the proposal, and the total number of the spectrum bands has increased from $N$ to $N+3$. The increase in the number of bands would cause an increasing number of the lossless bit rate. However, it could not be said that the difference of the lossless bit rate in the spectrum domain is a fatal in performance. The most valuable point of the Table 1 is a lossless bit rate of RGB data, which is one-eighth of the JPEG2000-MCT. In case that both lossless RGB data and lossless spectral data are required, the proposed method can provide both of the data at 7.179 bpppb but the conventional JPEG2000-MCT requires $0.784+6.820=7.604 \mathrm{bpppb}$. 
Fig. 5 shows a rate-distortion plot against bit rate on the RGB domain. The PSNR is infinity when lossless compression is performed in the graph. The proposed method encodes RGB component with high priority, the RGB image can be decoded with small distortion at low bit rate. In comparison, both the lossless bit rate and the distortion of the JPEG2000-MCT are inferior as compared with the proposed method. It would appear that the RGB component is scattered all around the code-stream in the case of JPEG2000-MCT.

The comparison of decoded MSI is shown in Fig. 6. We choose 3 points in the image (a) and observe 4 conditions, original, $0.1,0.7$ and 1.0 of the total bit rate. The first remarkable point is that the decoded MSI at $1.0 \mathrm{bpppb}$ which includes about $0.2 \mathrm{bpppb}$ residual data is almost identical to the original form. As mentioned earlier, residual data can contribute greatly to the improvement of the spectrum distortion. As another point to be focused, in the decoded MSI at 0.7 bpppb estimated from only the RGB data, the approximation accuracy of the spectrum varies depending on color. The shape of the spectrum in the background is closer to the original, but the shape in the colorful area is not improved compared with $0.1 \mathrm{bpppb}$. An increase in RGB data moves the estimated MSI close to the MSI reconstructed from lossless RGB data, but not to the original MSI.

\subsection{Performance of RGB Lossy Decoding}

The ineffective improvement of spectrum distortion while decoding of RGB components is a problem that needs to be solved, as seen in Sec. 3.1. For more improvement of the distortion, it is considered that the residual decoding should be started at earlier time. We propose one of the solutions for this problem and show 2 progressive orders in Fig. 7, "Lossless RGB mode" and "Lossy RGB mode". Note that the meaning of "Lossless" and "Lossy" is in only decoding. An encoded code-stream includes full of RGB code-stream in both modes. The "Lossless RGB mode" means the simple decoding as shown in Sec. 3.1. The "Lossy RGB mode" is a new progressive order, which is based on a concept that the residual decoding is conducted ahead of the decoding of an insignificant

Encoder

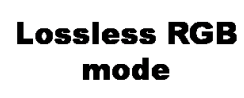
Lossy RGB
mode

\begin{tabular}{|c|c|}
\hline $\begin{array}{c}\text { RGB } \\
\text { Lossless }\end{array}$ & $\begin{array}{c}\text { Residual } \\
\text { Lossless }\end{array}$ \\
\hline
\end{tabular}

\begin{tabular}{|c|c|}
\hline $\begin{array}{c}\text { RGB } \\
\text { Lossless }\end{array}$ & $\begin{array}{c}\text { Residual } \\
\text { Lossless }\end{array}$ \\
\hline
\end{tabular}

Decoder

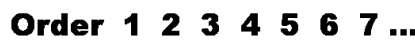

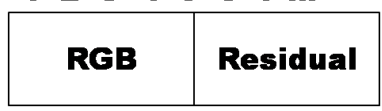

$12 \operatorname{xxxxx} 345 \ldots$

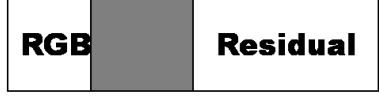

Figure 7. Decoding order.

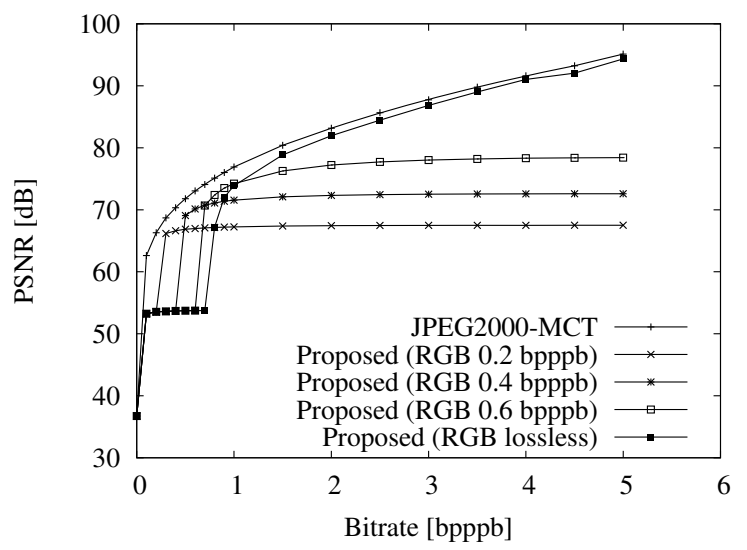

(a) Spectrum domain

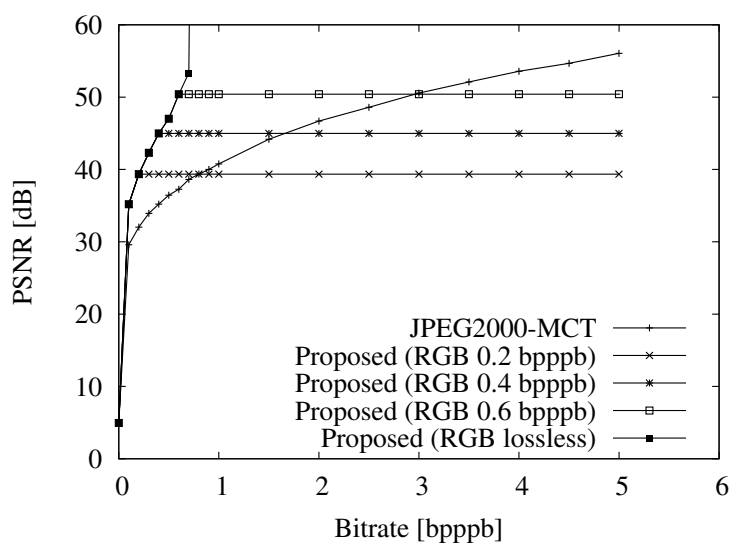

(b) RGB domain

Figure 8. Rate-Distortion plot depending on RGB rate. 


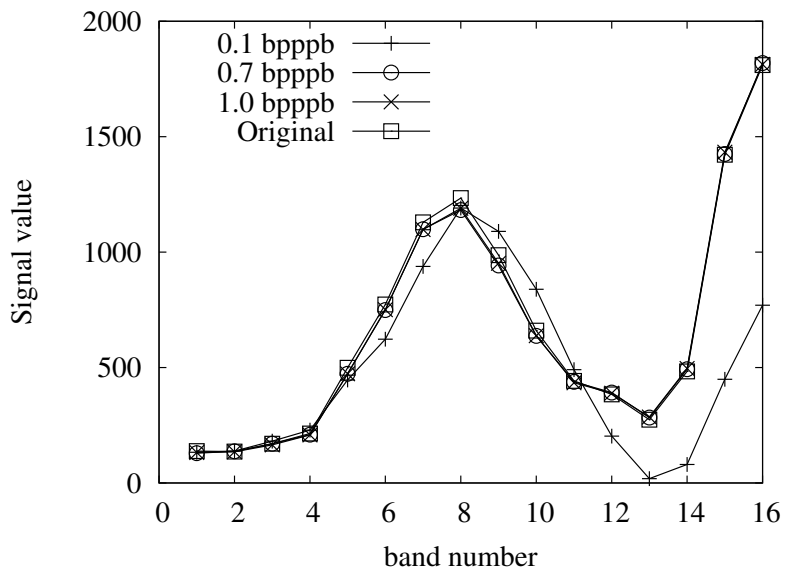

(a) Green $(200,160)$

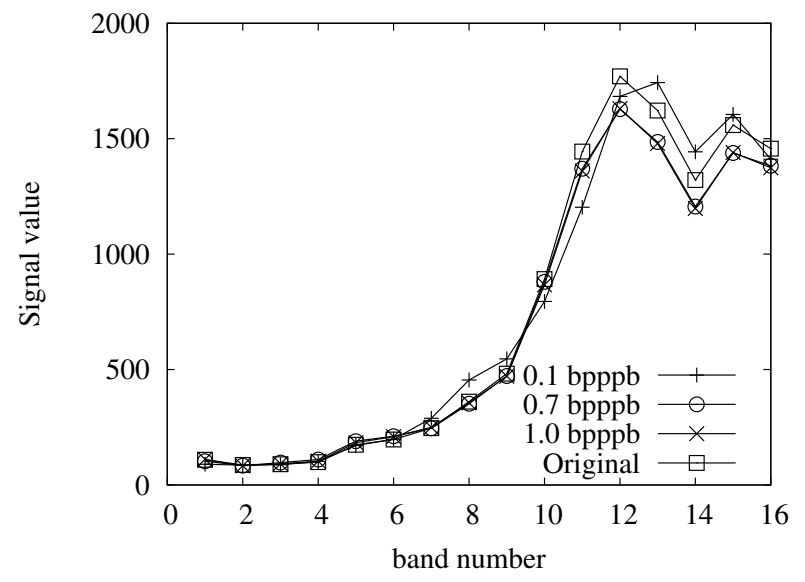

(b) Orange $(330,185)$

Figure 9. Decoded multispectral data with limited RGB data (0.2 bpppb) at each points.

RGB data. Although this mode can not provide a lossless RGB image, the spectrum distortion at low bit rate is expected to improve.

Fig. 8 shows a rate-distortion plots against bit rate on the spectrum and the RGB domain. The test image is used the same one in Fig. 3 (b). Consequently, the plots of "JPEG2000-MCT" and "RGB Lossless" in Fig. 8 are the exact same line in Fig. 4 and Fig. 5. Fig. 8 (a) indicates that the lossy RGB mode allows to reduce the spectrum distortion in the low bit rate because the residual decoding is started earlier than the lossless RGB mode. The plots show a clear improvement at the timing of adding residual data. The result means that the significant residual data can greatly reduce the spectrum distortion even if the RGB data is not fully decoded. From a practical perspective, the lossy RGB mode should be used to reduce the spectrum distortion in case that a system does not require a precise RGB image. By contrast, the RGB distortion increases because of the truncation of the RGB code-stream as shown in Fig. 8 (b). The lossless RGB mode should be used when a precise RGB image is required.

The reconstructed spectra are shown in Fig. 9. For the comparison between Fig. 9 (a) and Fig. 6 (c), the curve of the 0.7 bpppb is moved closer to the original by limiting the RGB bit rate. However, Fig. 9 (b) does not show the same trend as Fig. 9 (a). The result indicates that it is hard to converge the decoded spectrum to the true value because of the lack of RGB data. It is deemed desirable to use RGB 0.6 bpppb mode in the case not only for a precise RGB image, but also a precise reduction of spectrum at higher bit rate.

\section{CONCLUSION}

We have presented a lossy to lossless compression for multispectral image, while keeping the quality of RGB image for color reproduction. A compressed bit-string consists of RGB component and residual component, and the RGB components meet a requirement for sRGB and ITU-R BT.709. We estimate an original multispectral image from the RGB component on the encoder, and the difference between an original multispectral image and an estimated multispectral image is defined as the residual component. JPEG2000 with a multicomponent transform is used as a bit-coding of residual component. The proposed method is competitive to JPEG2000$\mathrm{MCT}$ at high bit rate in the spectrum domain. Experimental results in the RGB domain, excellent performances of the proposed are shown with respect to lossless and lossy bit rate. 


\section{REFERENCES}

[1] M. Yamaguchi, H. Haneishi, and N. Ohyama, "Beyond Red-Green-Blue(RGB): Spectrum-Based Color Imaging Technology," Journal of Imaging Science and Technology, Vol. 52, No. 1, Jan. 2008.

[2] P. L. Dragotti, G. Poggi, and A. R. P. Ragozini, "Compression of multispectral images by three-dimensional SPIHT algorithm," IEEE Transactions on Geoscience and Remote Sensing, vol. 38, no. 1, pp. 416-428, 2000.

[3] E. Christophe, C. Mailhes, and P. Duhamel, "Hyperspectral image compression: adapting SPIHT and EZW to anisotropic 3D wavelet coding," IEEE Transactions on Image Processing, vol. 17, issue 12, pp. 2334-2346, Dec. 2008.

[4] E. Christophe and W. A. Pearlman, "Three-Dimensional SPIHT Coding of Volume Images with Random Access and Resolution Scalability," Eurasip J. on Image and Video Processing, vol. 2008, Article ID 248905, 2008.

[5] T. Keusen, "Multispectral color system with an encoding format compatible with the conventional tristimulus model," J. Imaging Sci. Technol. 40(6), pp. 510-515, 1996.

[6] B. Hill, "Color capture, color management and the problem of metamerism: does multispectral imaging offer the solution?," Proc. SPIE, 3963, 2-14, 2000.

[7] S. Yu, Y. Murakami, T. Obi, M. Yamaguchi and N. Ohyama, "Multispectral Image Compression for High Fidelity Colorimetric and Spectral Reproduction," Journal of Imaging Science and Technology, Vol. 50, No. 1, pp. 64-72, Jan. 2006.

[8] ITU-T Rec. T.800-ISO/IEC JTC1/SC29/WG1 15444-1, "Information technology - JPEG2000 image coding system," Mar. 2001.

[9] S. Yu, Y. Murakami, T. Obi, M. Yamaguchi and N. Ohyama, "Multispectral Image Compression for Improvement of Colorimetric and Spectral Reproducibility by Nonlinear Spectral Transform," Optical Review, Vol. 13, No. 5, pp. 346-356, Sep. 2006.

[10] S. Takamura and Y. Yashima, "Multiband Video Signal Coding with H.264/AVC, MPEG-4 Studio Profile and Motion JPEG 2000 and Associated Signal Format," Journal of the Institute of Image Electronics Engineers of Japan, Vol.33, No.5, pp.792-801, 2004.

[11] IEC 61966-2-1, Multimedia systems and equipment - Colour measurement and management - Part 2-1: Colour management - default RGB colour space - sRGB, 1999.

[12] W. K. Pratt and C. E. Mancill, "Spectral estimation techniques for the spectral calibration of a color image scanner," Applied Optics, Vol.15, No.1, pp. 73-75, 1976.

[13] P. Hao and Q. Shi, "Matrix factorizations for reversible integer mapping," IEEE Transactions on Signal Processing, vol.49, no.10, pp.2314-2324, Oct 2001.

[14] LuraWave.jp2 Software Development Kit for C / C++ Version 1.12, 2009, https://www.luratech.com/. 